Case Report

\title{
Cerebral Arterial Air Embolism after Diagnostic Flexible Fiberoptic Bronchoscopy: A Case Report and Review of the Literature
}

\author{
Keita Maemura $\mathbb{D}^{1},{ }^{1}$ Hidenori Kage $\mathbb{D}^{1},{ }^{1}$ Hideaki Isago, ${ }^{1}$ \\ Hideyuki Takeshima, ${ }^{1}$ Kosuke Makita, ${ }^{1}$ Yosuke Amano $\mathbb{D},{ }^{1}$ Daiya Takai $\mathbb{D},{ }^{2}$ \\ Nobuya Ohishi, ${ }^{1}$ and Takahide Nagase ${ }^{1}$ \\ ${ }^{1}$ Department of Respiratory Medicine, The University of Tokyo Graduate School of Medicine, Tokyo, Japan \\ ${ }^{2}$ Department of Clinical Laboratory, The University of Tokyo Hospital, Tokyo, Japan \\ Correspondence should be addressed to Hidenori Kage; kageh-tky@umin.ac.jp
}

Received 4 January 2018; Accepted 26 March 2018; Published 7 May 2018

Academic Editor: Tun-Chieh Chen

Copyright (c) 2018 Keita Maemura et al. This is an open access article distributed under the Creative Commons Attribution License, which permits unrestricted use, distribution, and reproduction in any medium, provided the original work is properly cited.

\begin{abstract}
Cerebral arterial air embolism (CAAE) is an extremely rare complication of diagnostic flexible fiberoptic bronchoscopy, reported to occur once about every 103978 examinations. In all the eight cases of CAAE reported previously, the patients had undergone transbronchial lung biopsy (TBLB) or transbronchial needle aspiration (TBNA) prior to the onset of CAAE. Herein, we describe the case of a 77-year-old patient with double primary lung cancer who developed CAAE after bronchial curette cytology, which is considered to be less invasive than TBLB or TBNA. The patient was treated with supplemental oxygen, but paresis of the left upper arm and left spatial neglect remained. This is the first report of CAAE occurring after bronchial curettage during diagnostic flexible fiberoptic bronchoscopy.
\end{abstract}

\section{Introduction}

Arterial air embolism (AAE) is known to occur after scuba diving or as an iatrogenic complication. It is a well-known complication of CT-guided lung biopsy but occurs much less frequently as a complication of diagnostic flexible fiberoptic bronchoscopy. According to a nationwide survey of the complications of bronchoscopy conducted in 2010 in Japan, in which questionnaires were collected from 483 out of 538 facilities certificated by the Japan Society for Respiratory Endoscopy, AAE was reported at a frequency of about once every 103978 examinations $(0.00096 \%)$ [1]. This frequency is much lower than that reported in patients undergoing CTguided lung biopsy, in whom AAE is known to occur at a frequency of 0.06 to $0.45 \%[2,3]$. Of all types of $\mathrm{AAE}$, coronary AAE and cerebral AAE are the most serious and potentially fatal. Herein, we present a patient who developed cerebral arterial air embolism (CAAE) after diagnostic flexible fiberoptic bronchoscopy.

\section{Case Presentation}

A 77-year-old male patient with a smoking history of 55 packyears was referred to our hospital with an abnormality on the chest X-ray detected during his annual medical checkup (Figure 1).

Contrast-enhanced CT revealed a cavitary mass measuring $50 \mathrm{~mm}$ in diameter in the right lower lobe, a mass measuring $34 \mathrm{~mm}$ in diameter in the left lower lobe, and an enlarged left hilar lymph node (\#11) (Figure 1). No metastatic lesions were detected. Based on these findings, the patient was strongly suspected as having a double primary lung cancer.

At the first admission, flexible fiberoptic bronchoscopy was performed, and the cytological findings in endobronchial ultrasound-guided transbronchial needle aspiration (EBUSTBNA) specimens obtained from the left hilar lymph node were classified as class V, squamous cell carcinoma. After the examination, slight left pneumothorax was noted, which improved spontaneously without additional treatment. 


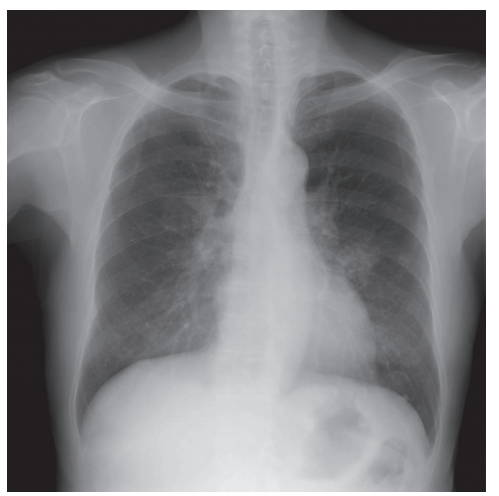

(a)

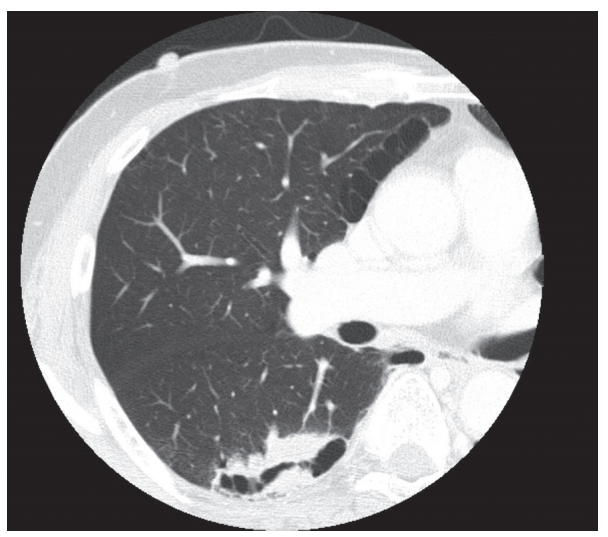

(c)

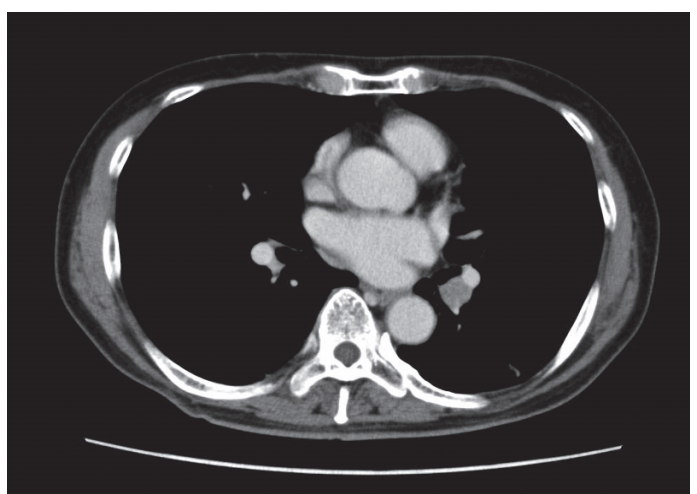

(b)

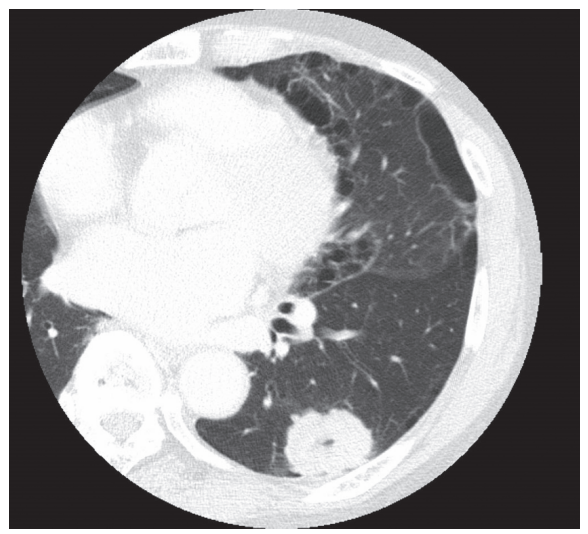

(d)

FIGURE 1: Radiologic examinations at the first visit to our hospital. (a) Chest X-ray. (b-d) Contrast-enhanced CT scan. (b) The left hilar lymph node. (c) The mass in the right lung. (d) The mass in the left lung.

At the second admission, flexible fiberoptic bronchoscopy was performed again under conscious sedation achieved by intravenous injection of $1.5 \mathrm{mg}$ of midazolam. The patient was placed in the left lateral position and the cytological findings in the specimens obtained by curettage and instillation of saline into the right $\mathrm{B}^{6}$ a were classified as class III. The patient had a little bleeding and coughing during the examination. After the examination, $0.2 \mathrm{mg}$ of flumazenil was injected intravenously; however, the patient remained drowsy. Other vital signs were normal. When he became more alert three hours after the examination, he was found to have slurred speech, left hemiplegia, and left spatial neglect. The National Institute of Health Stroke Scale (NIHSS) score was 21. Emergency head CT revealed obscuring of the corticomedullary junction in the brain region supplied by the right middle cerebral artery (MCA) (Figure 2), and cerebral infarction was suspected. Reconstruction of the CT images using the mediastinal window settings revealed scattered air bubbles in the MCA region, based on which the patient was definitively diagnosed as having developed CAAE (Figure 2). Instead of hyperbaric oxygen $\left(\mathrm{HBO}_{2}\right)$ therapy, which was not available at our hospital, we administered normobaric oxygen $\left(\mathrm{NBO}_{2}\right)$ therapy $(10 \mathrm{~L} / \mathrm{min}$ of oxygen delivered via a nonrebreathing mask). The following day, the patient received fosphenytoin and levetiracetam for clonic convulsion extending from his left face to the upper arm. A repeat head CT showed disappearance of the air bubbles but emergence of cerebral edema, which improved over the following six days. While the patient was eventually able to walk using a four-legged cane, paresis of the left upper arm and left spatial neglect remained, and the Eastern Cooperative Oncology Group (ECOG) performance status was estimated to be 3 . We therefore discontinued any further investigation or treatment of the lung cancer. Approximately four months after the second admission, the patient was transferred to a hospice near his hometown.

\section{Discussion}

This is the ninth reported case in the literature of CAAE developing after diagnostic flexible fiberoptic bronchoscopy. The profiles of the reported patients, the examination procedures undertaken, the treatments, and the outcomes are shown in Table 1 [4-10]. TBLB or TBNA was performed prior to the development of CAAE in all the previously reported cases. Our case was the first and only one to develop CAAE after bronchial curettage (strictly speaking, instillation of saline into the lesion of small volume in $S^{6}$ a may have induced CAAE by increasing the pressure of the peripheral airway). As curettage is a minimally invasive procedure, this case serves to underscore the importance for pulmonologists to be aware 


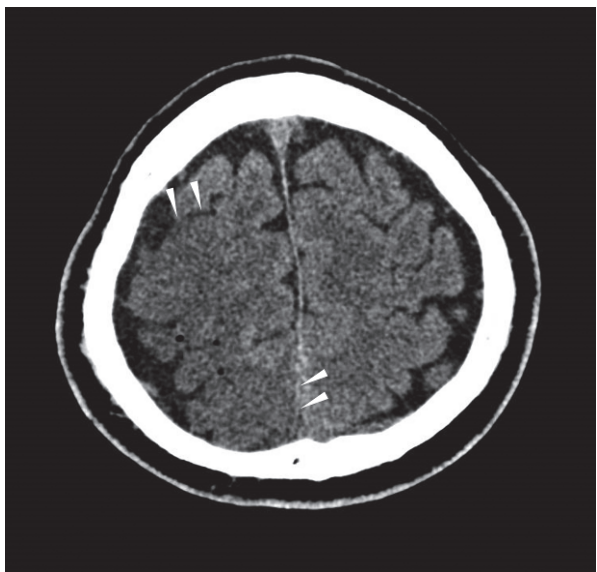

(a)

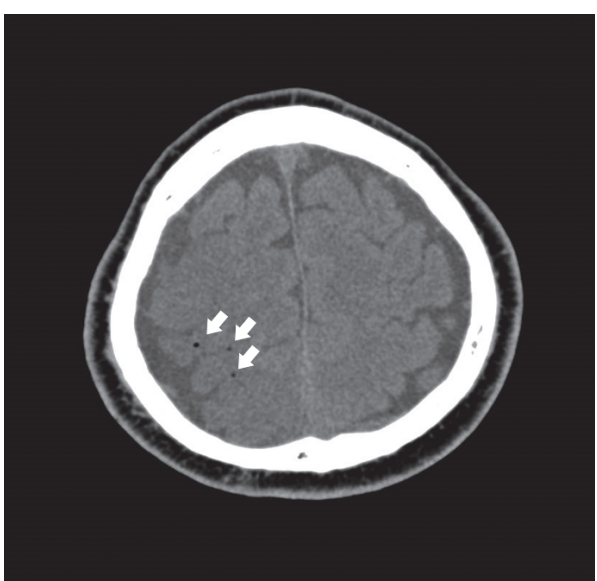

(b)

FIGURE 2: The same slice of head CT on the day of the onset of CAAE. The arrowheads show the obscureness of the corticomedullary junction. The arrows show the embolization of air bubbles. (a) Displayed with normal brain window settings (window width, 80 Hounsfield Units (HU); window level, $40 \mathrm{HU}$ ). (b) Displayed with mediastinal window settings (window width, $250 \mathrm{HU}$; window level, $40 \mathrm{HU}$ ).

that any procedure that can cause bleeding could result in CAAE.

In patients undergoing CT-guided lung biopsy, location of the lesion in the lower lobe, occurrence of parenchymal hemorrhage, and use of a larger biopsy needle are all significant risk factors for the development of CAAE [3]. In contrast, the frequency of CAAE developing after diagnostic flexible fiberoptic bronchoscopy is too low to allow a statistically reasonable discussion of the risk factors for CAAE in patients undergoing bronchoscopy. However, we consider some shared factors between our case and some of the other eight cases as possible rick factors for CAAE occurring after bronchoscopy. In patients with chronic obstructive pulmonary disease (four cases, 44\%), air from enlarged airspaces may easily flow into injured vessels. A cavity inside the mass (two cases, 22\%) may also be a risk factor for the same reason. The diagnostic procedures were performed for lesions in the upper lobe in five (56\%) cases and in $S^{6}$ in three $(33 \%)$ cases. No logical explanation for lesion location as a risk factor for CAAE can be provided based on this distribution; when the patient is in the sitting position, the lower pressure of the pulmonary veins in the upper lung field may lead to increased susceptibility to the development of CAAE, but this explanation would not be valid for patients in the supine position or lateral position, that is, during bronchoscopy. Before the onset of CAAE, the patient was laid in the left lateral position in two (22\%) cases. No previous report has indicated the left lateral position as a risk factor for CAAE occurring during bronchoscopy or CT-guided lung biopsy, although air bubbles entering the left side of the heart may easily float up along the right side of the aortic wall into the right common carotid artery in this position, resulting in infarction of the right hemisphere. Our case fulfilled all of these four potential risk factors. Another factor to consider is bleeding during the procedure in two $(22 \%)$ cases, because injury to the vessel wall may allow air to enter the vessel. Moreover, bleeding can induce coughs, which increase the intrabronchial pressure, leading to air embolism.

Definitive diagnosis of CAAE can be made when a CT image reveals air bubbles in the cerebral artery, but more subtle changes cannot be visualized on CT images. Therefore, some argue that visualization of air bubbles is not essential for the diagnosis of CAAE [11]. In one of the reported cases, no air bubbles could be visualized on the CT images, and the diagnosis was made by exclusion, that is, after confirming that there was no evidence of hypotension, hypoglycemia, or other causes of brain infarction [7]. Distribution of the ischemic lesions at the corticomedullary junction on brain magnetic resonance imaging (MRI) and refractory convulsions with little response to benzodiazepine administration are reported as findings that lend support to the diagnosis of CAAE $[7,12]$.

It is difficult to consider AAE in the differential diagnosis of complications occurring during bronchoscopy, because of its rarity. Moreover, we do not routinely perform CT to detect air bubbles after bronchoscopy. Therefore, asymptomatic $\mathrm{AAE}$ and self-limited air entry into the left atrium may have been missed. Although the frequency of AAE is likely lower in bronchoscopy than in CT-guided lung biopsy, the actual incidence of AAE in bronchoscopy may be higher than previously thought. For instance, some proportion of patients with cardiovascular events associated with bronchoscopy (71 cases, $0.068 \%$ in the previous survey [1]) may include those with subtle coronary AAE with normal coronary angiography, or cerebral AAE with normal brain CT. In our case, we detected CAAE in images reconstructed with mediastinal window settings, which helped in better visualization of the air bubbles. Figures 2(a) and 2(b) represent the same slice, but the air bubbles are more easily visualized in Figure 2(b). A recent paper in a radiological journal also pointed out the usefulness of window setting adjustments for visualizing air bubbles [13].

The recommended treatment for CAAE is $\mathrm{HBO}_{2}$ therapy. The US Navy manual for scuba diving recommends 


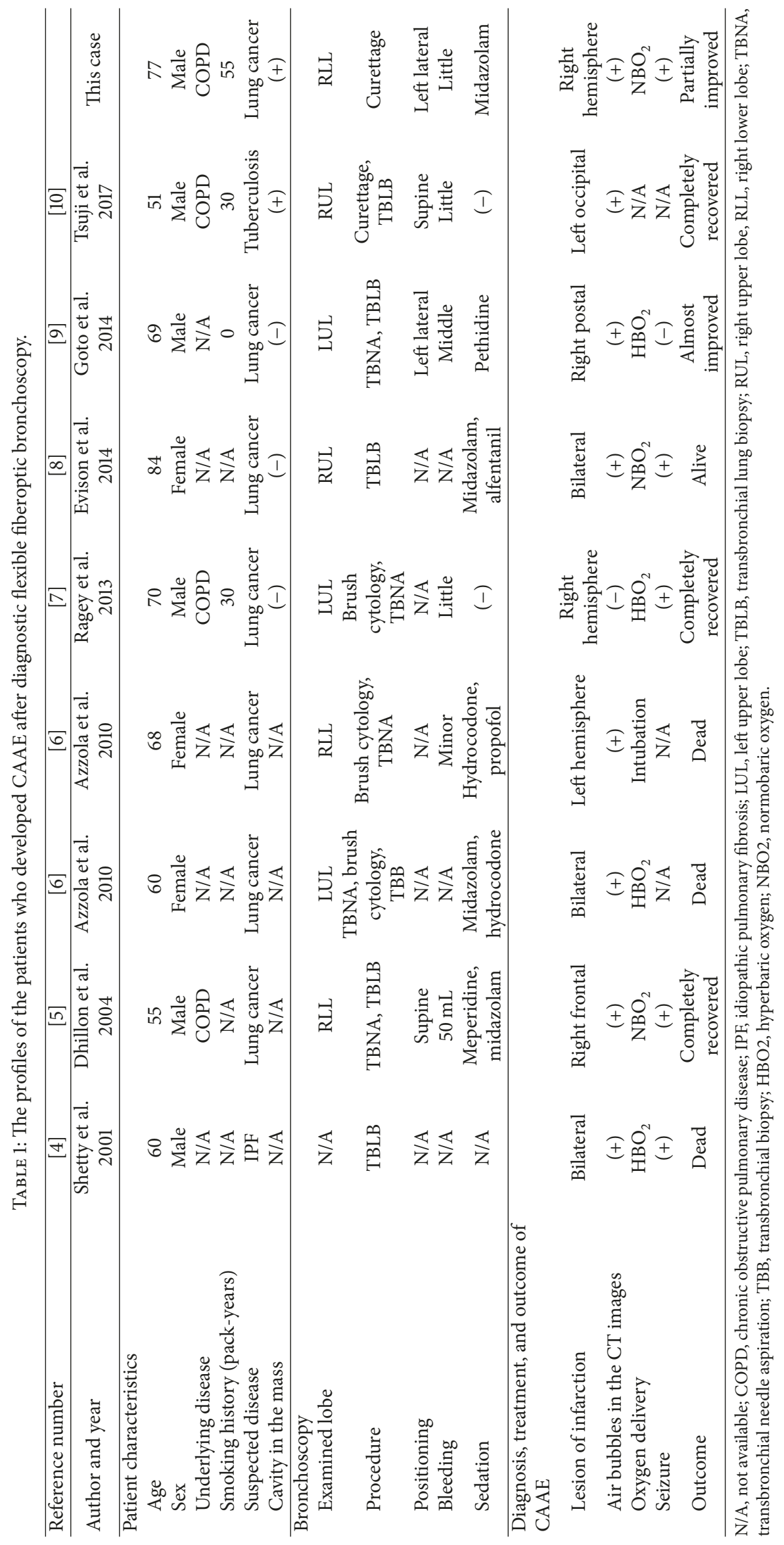


recompression to a pressure equivalent to that at a water depth of 60 feet (approximately 2.8 atmospheric pressures) immediately upon suspicion of AAE [14]. A definitive diagnosis is not essential for starting the therapy. The first rationale for using $\mathrm{HBO}_{2}$ therapy is that the hyperbaric environment reduces the volume of air bubbles, in accordance with Boyle's law (the gas volume is inversely proportional to the gas pressure). The second is that the low partial pressure of nitrogen in the blood makes it easy for the nitrogen gas in the bubbles to dissolve into the blood, in accordance with Fick's law (the diffusive flux is proportional to the concentration gradient). The third is that the high partial pressure of oxygen in the blood increases oxygen delivery to the damaged tissues [11]. When there is no access to a recompression chamber or $\mathrm{HBO}_{2}$ therapy is contraindicated, $\mathrm{NBO}_{2}$ therapy can be administered instead $[5,8,11]$. This satisfies the second and the third rationales for $\mathrm{HBO}_{2}$ therapy and has been shown to shorten the time taken for complete resolution of the air bubbles in dog studies [15].

In summary, we present the first case of CAAE developing as a complication of bronchial curettage performed during diagnostic flexible fiberoptic bronchoscopy. All pulmonologists should be aware of the possible occurrence of this complication after bronchoscopy, and should ensure prompt administration of supplemental oxygen, preferably $\mathrm{HBO}_{2}$ therapy, upon suspicion of AAE.

\section{Conflicts of Interest}

The authors declare that they have no conflicts of interest.

\section{References}

[1] F. Asano, M. Aoe, Y. Ohsaki et al., "Deaths and complications associated with respiratory endoscopy: A survey by the Japan Society for Respiratory Endoscopy in 2010," Respirology, vol. 17, no. 3, pp. 478-485, 2012.

[2] N. Tomiyama, Y. Yasuhara, Y. Nakajima et al., "CT-guided needle biopsy of lung lesions: a survey of severe complication based on 9783 biopsies in Japan," European Journal of Radiology, vol. 59, no. 1, pp. 60-64, 2006.

[3] H. Ishii, T. Hiraki, and H. Gobara, "Risk factors for systemic air embolism as a complication of percutaneous CT-guided lung biopsy: multicenter case-control study," CardioVascular and Interventional Radiology, vol. 37, no. 5, pp. 1312-1320, 2014.

[4] P. G. Shetty, G. M. Fatterpekar, S. Manohar, V. Sujit, J. Varsha, and U. Zarir, "Fatal cerebral air embolism as a complication of transbronchoscopic lung biopsy: a case report," Journal of Medical Imaging and Radiation Oncology, vol. 45, no. 2, pp. 215217, 2001.

[5] S. S. Dhillon, A. Agrawal, and A. B. Gorin, "Cerebral arterial air embolism after transbronchial lung biopsy: a case report and review of literature," Journal of Bronchology \& Interventional Pulmonology, vol. 11, no. 2, pp. 125-127, 2004.

[6] A. Azzola, C. Von Garnier, P. N. Chhajed, U. Schirp, and M. Tamm, "Fatal cerebral air embolism following uneventful flexible bronchoscopy," Respiration, vol. 80, no. 6, pp. 569-572, 2010.
[7] S. P. Ragey, P. Garnier, and J.-M. Vergnon, "Complete resolution of cerebral air embolism secondary to a transbronchial needle aspiration," Respiration, vol. 86, no. 6, pp. 504-507, 2013.

[8] M. Evison, P. A. J. Crosbie, R. Bright-Thomas, M. Alaloul, and R. Booton, "Cerebral air embolism following transbronchial lung biopsy during flexible bronchoscopy," Respiratory Medicine Case Reports, vol. 12, pp. 39-40, 2014.

[9] H. Goto, M. Nakao, F. Ohyanagi et al., "A case of cerebral air embolism following bronchoscopy," The Journal of the Japan Society for Respiratory Endoscopy, vol. 36, no. 6, pp. 649-655, 2014.

[10] T. Tsuji, S. Sonobe, T. Koba, T. Maekura, N. Takeuchi, and K. Tachibana, "Systemic air embolism following diagnostic bronchoscopy," Internal Medicine, vol. 56, no. 7, pp. 819-821, 2017.

[11] C. M. Muth and E. S. Shank, "Gas embolism," The New England Journal of Medicine, vol. 342, no. 7, pp. 476-482, 2000.

[12] T. Hodics and I. Linfante, "Cerebral air embolism," Neurology, vol. 60, no. 1, p. 112, 2003.

[13] N. Zakhari, M. Castillo, and C. Torres, "Unusual cerebral emboli," Neuroimaging Clinics of North America, vol. 26, no. 1, pp. 147-163, 2016.

[14] Direction of Commander and Naval Sea Systems Command, U.S. Navy Diving Manual, Utah State University, 2008, http:// www.usu.edu/scuba/navy_manual6.pdf.

[15] D. Annane, G. Troché, F. Delisle et al., "Effects of mechanical ventilation with normobaric oxygen therapy on the rate of air removal from cerebral arteries," Critical Care Medicine, vol. 22, no. 5, pp. 851-857, 1994. 


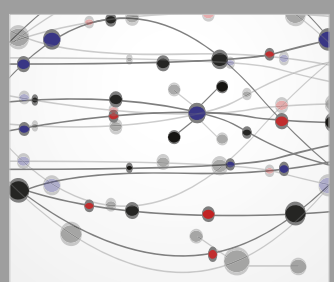

The Scientific World Journal
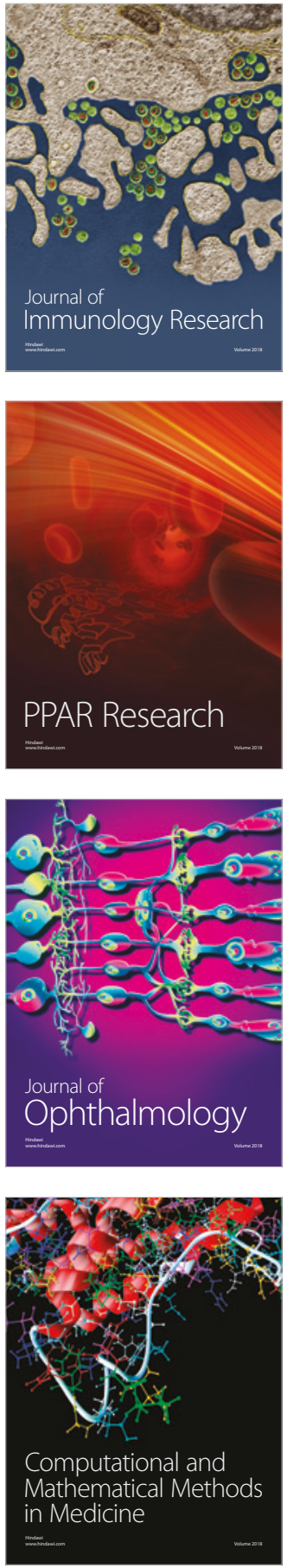

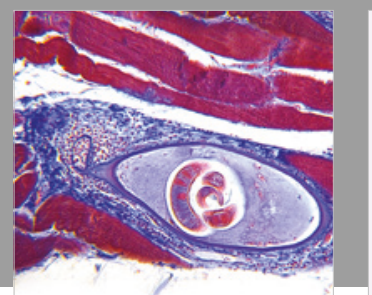

Gastroenterology Research and Practice

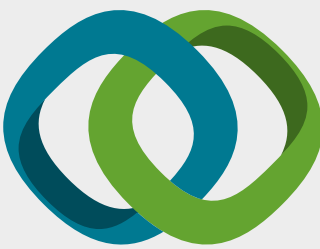

\section{Hindawi}

Submit your manuscripts at

www.hindawi.com
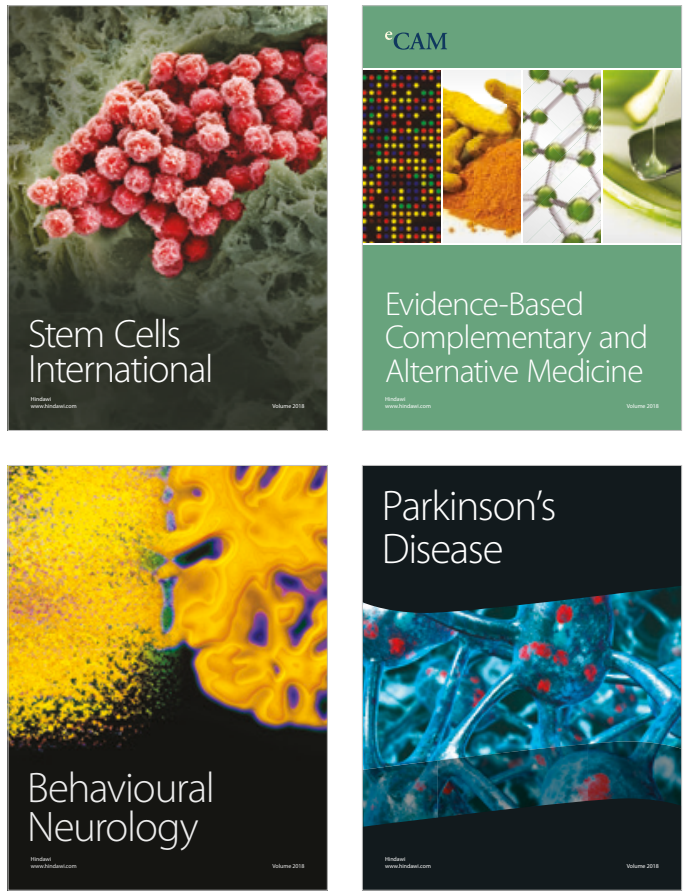

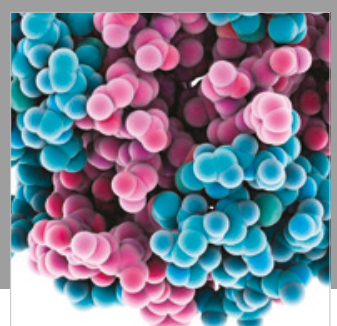

ournal of

Diabetes Research

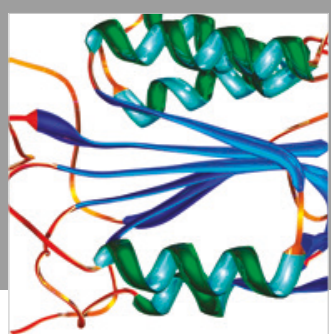

Disease Markers
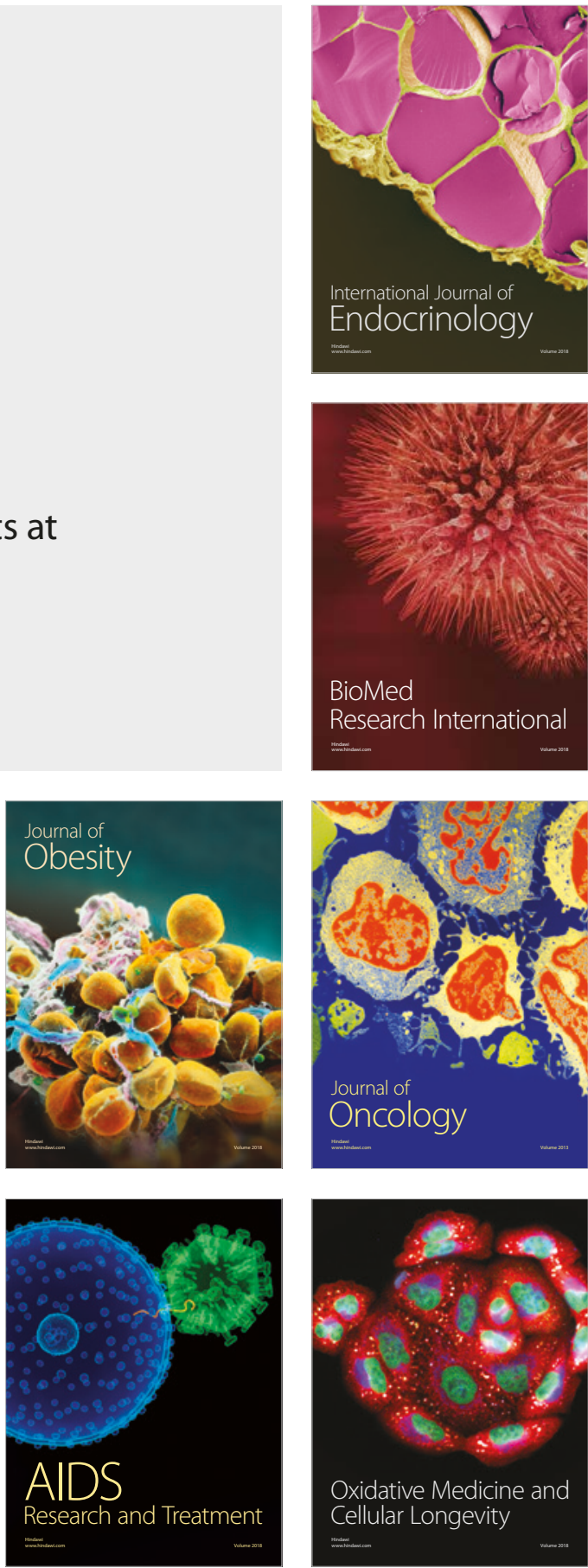